\title{
An unusual Dicer-like1 protein fuels the RNA interference pathway in Trypanosoma brucei
}

\author{
HUAFANG SHI, ${ }^{1}$ CHRISTIAN TSCHUDI, ${ }^{1,2}$ and ELISABETTA ULLU ${ }^{1,3}$ \\ ${ }^{1}$ Department of Internal Medicine, Yale University Medical School, New Haven, Connecticut 06536-0812, USA \\ ${ }^{2}$ Department of Epidemiology \& Public Health, Yale University Medical School, New Haven, Connecticut 06536-0812, USA \\ ${ }^{3}$ Department of Cell Biology, Yale University Medical School, New Haven, Connecticut 06536-0812, USA
}

\begin{abstract}
RNA interference (RNAi) is an evolutionarily conserved gene-silencing pathway that is triggered by double-stranded RNA (dsRNA). Central to this pathway are two ribonucleases: Dicer, a multidomain RNase III family enzyme that initiates RNAi by generating small interfering RNAs (siRNAs), and Argonaute or Slicer, an RNase $\mathbf{H}$ signature enzyme that affects cleavage of mRNA. Previous studies in the early diverging protozoan Trypanosoma brucei have established a key role for Argonaute 1 in RNAi. However, the identity of Dicer has not been resolved. Here, we report the identification and functional characterization of a $T$. brucei Dicer-like enzyme (TbDcl1). Using genetic and biochemical approaches, we provide evidence that TbDcl1 is required for the generation of siRNA-size molecules and for RNAi. Whereas Dicer and Dicer-like proteins are endowed with two adjacent RNase III domains at the carboxyl terminus (RNase IIla and RNase IIIb), the arrangement of these two domains is unusual in TbDcl1. RNase IIla is close to the amino terminus, and RNase IIIb is located approximately in the center of the molecule. This domain organization is specific to trypanosomatids and further illustrates the variable structures of protozoan Dicer-like proteins as compared to fungal and metazoan Dicer.
\end{abstract}

Keywords: RNA interference; Dicer; RNase III; trypanosome

\section{INTRODUCTION}

The execution of RNA degradation (RNA interference, RNAi) triggered by homologous double-stranded RNA (dsRNA) requires the assistance of two endoribonucleases, namely, Dicer, an RNase III family enzyme, and Slicer, a member of the Argonaute (Ago) protein family, an RNase H-type enzyme (Tomari and Zamore 2005; Zamore and Haley 2005). Dicer (Dcr) is required for the biogenesis of two classes of small RNAs: the small interfering RNAs (siRNAs), which are processed from long dsRNA, and the microRNAs (miRNAs), which are processed from premiRNAs. siRNAs (and in certain instances miRNAs) are then loaded into Slicer, which is the endonuclease of the RNA-induced silencing complex (RISC) and functions by cleaving the mRNA across the duplex formed with the guide small RNA strand (Liu et al. 2004). So far only a subset of Ago proteins has been demonstrated to be endowed with

Reprint requests to: Elisabetta Ullu, Department of Internal Medicine, Yale University Medical School, 295 Congress Avenue, New Haven, CT 06536-0812, USA; e-mail: elisabetta.ullu@yale.edu; fax: (203) 785-7329.

Article published online ahead of print. Article and publication date are at http://www.rnajournal.org/cgi/doi/10.1261/rna.246906.
Slicer activity, including human Ago2 (Liu et al. 2004; Meister et al. 2004; Rivas et al. 2005), Arabidopsis thaliana Ago1 (Baumberger and Baulcombe 2005), Drosophila Ago2 (Miyoshi et al. 2005), and Trypanosoma brucei Ago1 (Shi et al. 2004b, 2006).

Dicer and Dicer-like (Dcl) proteins belong to the RNase III family of dsRNA-specific endoribonucleases (Carmell and Hannon 2004). Members of this family are quite diverse both in size and domain structure. The simplest RNase III proteins are mostly of prokaryotic origin and are characterized by a single RNase III domain followed by a dsRNA binding domain (dsRBD). The domain structure becomes more elaborate in Drosha and homologs, which have been identified in flies, worms, humans, and mice. These proteins have two neighboring RNase III domains, a dsRBD and an amino-terminal extension of $\sim 900$ amino acids. Finally, Dicer and Dicer-like proteins are large multidomain proteins between 135 and $200 \mathrm{kDa}$ (Bernstein et al. 2001; Carmell and Hannon 2004; Cerutti and CasasMollano 2006) and typically contain an amino-terminal DExH RNA helicase/ATPase domain, followed by a domain of unknown function (DUF283), a PAZ domain, which anchors the $3^{\prime}$-end of the guide siRNA, two neighboring RNase III domains (RNase IIIa and RNase IIIb), and 
a dsRBD. Although this overall organization is maintained in animals, plants, and fungi, it has also become apparent that this domain architecture does not apply to all organisms (Carmell and Hannon 2004; Cerutti and CasasMollano 2006). For instance, Tetrahymena thermophila Dcl1 (Mochizuki and Gorovsky 2005), which is required for the production of siRNA-like scan RNAs, has only the two neighboring RNase III domains and a dsRBD. Giardia intestinalis Dicer (Macrae et al. 2006) consists of a PAZ domain, a highly divergent DUF283 motif, and two adjacent RNase III domains. Moreover, fission yeast Dcr1 and $A$. thaliana Dcr4 lack a recognizable PAZ domain, and there are other Dicers in which the DUF283 motif appears to be absent. Thus, throughout eukaryotic evolution, there has been a great diversification in the domain structure of Dicer-like proteins. To date, the only universal feature of this gene family is the presence of two neighboring RNase III domains located at the carboxyl terminus.

Although Dicer and Dicer-like proteins are commonly large, the Giardia enzyme has provided an exception to this rule. Indeed, Giardia Dicer is the smallest Dicer identified to date, consisting of 756 amino acids, which is less than half the size of human Dicer (Macrae et al. 2006). Nevertheless, recombinant Giardia Dicer is functional in vitro, and complementation of Schizosaccharomyces pombe lacking Dicer demonstrated that the Giardia enzyme is sufficient to function as Dicer in vivo. Importantly, the recent crystal structure of Giardia Dicer (Macrae et al. 2006), in combination with mutagenesis studies of recombinant human Dicer (Zhang et al. 2004), has provided the structural and functional basis for the current model for dsRNA cleavage by Dicer. In this model the RNase IIIa and RNase IIIb domains form an intramolecular dimer with a single processing site, while the PAZ domain binds to the end of dsRNA (Zhang et al. 2004; Macrae et al. 2006). Thus, Dicer is thought to function as a molecular ruler that recognizes dsRNA and cleaves at a fixed distance from the end. The variability in the size of siRNAs, ranging from 21 to 30 nucleotides (nt) in different organisms, is most likely accounted for by differences in structural features of Dicers.

Although recombinant human (Provost et al. 2002; Zhang et al. 2002) and Giardia (Macrae et al. 2006) Dicers function in vitro without the assistance of other proteins, in vivo the metazoan enzymes are involved in complex and, most likely, dynamic interactions. For instance, human Ago2 (Slicer) binds to Dicer directly (Tahbaz et al. 2004), possibly regulating its activity, and dsRNA binding proteins like Drosophila R2D2 (Liu et al. 2003) and the human immunodeficiency virus transactivating response RNAbinding protein (TRBP) (Chendrimada et al. 2005; Haase et al. 2005) are thought to assist in the transition of siRNAs from Dicer to RISC. Furthermore, there is evidence that Drosophila Dcr2 plays a role in RISC assembly and forms an essential component of a large mRNA-cleaving complex known as "holo-RISC" (Lee et al. 2004; Pham et al. 2004).
Thus, the current evidence supports a role of Dicer at early, as well as at later stages in the RNAi pathway.

In T. brucei, a parasitic protozoan of early evolutionary origin, RNAi is a constitutive pathway that appears to be dedicated to down-regulation of endogenous retroposon transcripts (Djikeng et al. 2001; Shi et al. 2004a) and has been proposed to have a role in chromosome segregation (Durand-Dubief and Bastin 2003). Whereas the identification of the TbAgo1/Slicer gene was quite straightforward due to the conservation of signature motifs of the Ago protein family (Durand-Dubief and Bastin 2003; Shi et al. 2004a), the discovery of a Dicer homolog has lagged behind, because database mining failed to identify a T. brucei protein with two neighboring RNase III domains. On the other hand, endogenous and transgene-derived siRNAs (Djikeng et al. 2001), as well as an in vitro Dicer-like activity (Best et al. 2005), have been characterized in T. brucei. The apparent absence of a protein with two RNase III domains has led us and others (Cerutti and Casas-Mollano 2006) to speculate that either the T. brucei genome is incomplete, that the trypanosome Dicer may be prokaryotic-like with a single RNase III domain, or that its sequence has diverged beyond recognition by primary sequence searches.

Here we report the identification and functional characterization of the T. brucei Dicer-like1 (TbDcl1) protein, which is required for processing dsRNA into siRNA-size molecules and for RNAi.

\section{RESULTS}

\section{Identification of a candidate Dicer homolog in $T$. brucei}

In order to identify potential Dicer gene candidates, we surveyed the $T$. brucei GeneDB database for proteins containing an annotated RNase III domain and performed BLAST searches with the Aquifex aeolicus RNase III domain. This approach returned eight open reading frames (ORFs), each containing a single predicted RNase III domain. Four (accession nos. Tb10.70.1580, Tb10.70.1750, Tb11.03.0180, and $\mathrm{Tb} 927.1 .1690)$ were previously identified as components of the editosome (the mitochondrial RNA editing complex) (Panigrahi et al. 2006), two (accession nos. Tb11.01.0150 and Tb11.46.0012) were annotated as hypothetical proteins conserved in Leishmania major and Trypanosoma cruzi, and the remaining two appeared to be only present in the T. brucei genome. Since both L. major and T. cruzi are RNAi negative (Robinson and Beverley 2003; DaRocha et al. 2004), we concentrated our attention on the two T. bruceispecific ORFs (Fig. 1A). These proteins were named according to the predicted number of amino acids as Tb1648 (accession no. Tb927.8.2370) and Tb948 (accession no. Tb927.3.1230). Further database mining revealed homologs of Tb1648 in the unfinished genomes of two African 


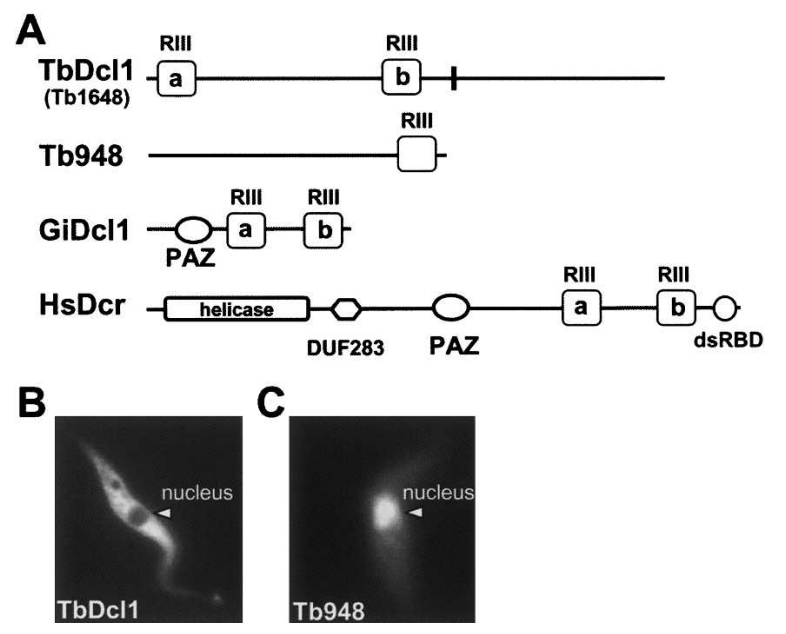

FIGURE 1. (A) Schematic representation of the domain structure of TbDcl1 (Tb1648), Tb948, G. intestinalis Dcl1 (GiDcl1), and human Dicer (HsDcr). The drawing is approximately to scale. A vertical bar in TbDcl1 indicates the approximate position of mutation D966G. The relative positions of the RNAse III (RIII) a and b motifs are indicated for each protein, as well as the PAZ, DUF283, and dsRBD. $(B, C)$ Cellular localization of Tb1648 and Tb948. Live procyclic trypanosomes, transiently expressing Tb1648-gfp or Tb948-gfp, were examined by fluorescence microscopy. A white arrowhead points to the nucleus.

trypanosomes that are closely related to $T$. brucei, namely, Trypanosoma congolense (congo592a12.q1k_6) and Trypanosoma vivax (tviv838h12.p1k_2). Importantly, T. congolense has been experimentally shown to have a functional RNAi pathway (Inoue et al. 2002), whereas T. vivax has a homolog of the TbAgol gene (tviv897e09.p1k_2), strongly suggesting that this organism is RNAi competent. In addition, a homolog of Tb948 was evident in T. congolense (congo347e08.q1kw_2).

To begin to address the function of the Tb1648 and Tb948 proteins, we determined the cellular localization by generating fusions with the green fluorescent protein (gfp) and expressing them transiently in cultured procyclic trypanosomes. This showed that Tb1648-gfp was predominantly cytoplasmic (Fig. 1B), which is the cellular localization of the RNAi machinery in T. brucei (Shi et al. 2004a), whereas Tb948-gfp was enriched in the nucleus (Fig. 1C). Taken together with the indication that the 1648 protein appeared to be restricted to RNAi-positive trypanosomatids, we decided to characterize Tb1648 in more detail. Intriguingly, there also appeared to be a correlation between the presence of the 948 protein and an RNAi machinery, and the function of this polypeptide is currently under study.

As mentioned above, the initial search exposed only one RNase III domain in the T. brucei 1648 protein located approximately in the middle of the protein (Fig. 1A). However, using the T. vivax protein to search the conserved domain database at NCBI with an expected threshold of 1 revealed an additional RNase III domain located close to the amino terminus. This domain also became apparent in the $T$. brucei protein when the expect threshold was increased to 100 . The identification of the amino terminus of TbDcll was based on the following observations. First, there are three stop codons and no other methionines upstream of the methionine identified as position 1 . Second, the similarity between the Dcll genes of T. brucei, $T$. vivax, and $T$. congolense does not extend upstream of position 1. However, the searches mentioned above did not recognize any other features of Dicer or Dicer-like enzymes, namely, the helicase domain, the DUF283 motif, the PAZ domain, or the dsRBD. Following the current nomenclature, the RNase III domains in the trypanosome proteins close to the amino terminus and in the center of the protein are referred to as RNase IIIa and RNase IIIb, respectively. Figure 2A shows the CLUSTALW alignment of the trypanosome RNase III-like domains with a representative sample of RNase III domains, including the A. aeolicus

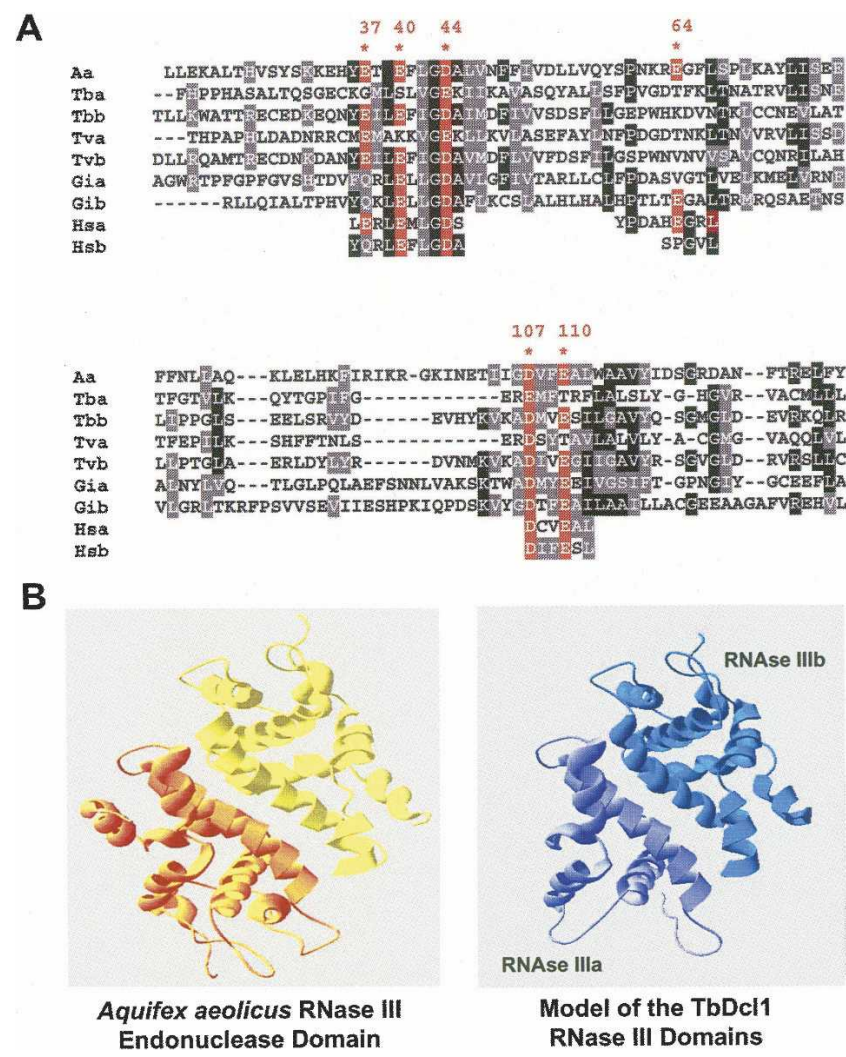

FIGURE 2. (A) CLUSTALW alignment of the A. aeolicus (Aa) RNase III domain with the RNase IIIa and IIIb motifs of TbDcll (Tba and Tbb), of the T. vivax Dcll homolog (Tva and Tvb), of G. intestinalis Dcll (Gia and Gib), and relevant sequences of human Dicer RNase IIIa and RNase IIIb, as described in Zhang et al. (2004). Numbers above the alignment refer to the A. aeolicus RNase III and indicate conserved acidic residues among bacterial RNase III (Gan et al. 2006). (B) Ribbon diagram of the secondary structure of A. aeolicus (Blaszczyk et al. 2001) RNase III (left panel) and of the predicted model of the RNase IIIa and IIIb motifs of TbDcll (right panel). 
RNase III domain. The crystal structure of $A$. aeolicus RNase III revealed the catalytic domain dimer to contain two clusters of six acidic side chains, namely, E37, E40, and D44, and E64, D107, and E110 (Blaszczyk et al. 2001), which are highly conserved among prokaryotic RNase III proteins and are indicated by asterisks in Figure 2A. The RNase IIIb domain of both the T. vivax and T. brucei 1648 proteins showed the presence of acidic residues at the equivalent positions of the $A$. aeolicus protein, except for the E64 residue, which is also not conserved in the G. intestinalis RNase IIIa and the human RNase IIIb domains. In contrast, in the T. brucei 1648 RNase IIIa domain, only the acidic residues at positions 44 and 107 were evident. Instead, in the T. vivax RNase IIIa domain, acidic residues were found at positions 37,44 , and 107 . To further probe the relationship between Tb1648 and RNase III, we performed three-dimensional structure modeling of the trypanosomatid RNase III domains. By means of the alignment interface of SWISS-MODEL, the $A$. aeolicus RNase III crystal structure was used as a template to generate a model for the Tb1648 RNase III domains (Fig. 2B). The model has many structural similarities with the prototypical structure of $A$. aeolicus RNase III (Blaszczyk et al. 2001), including the potential of the two RNase III domains to dimerize.

In conclusion, $\mathrm{Tb} 1648$ (herein referred to as TbDcl1) was found to be the most likely candidate for a trypanosome Dicer-like protein, because it appears to be specific to the genomes of RNAi-positive trypanosomes, is localized in the cytoplasm, has two RNase III-like domains, and the modeled structure is similar to that of prokaryotic RNase III.

\section{Affinity-purified TbDcl1 can dice dsRNA to siRNA-size fragments}

If TbDcll is indeed the trypanosome Dicer homolog, we expect that this protein will dice dsRNA into siRNA-size molecules of 24-26 nt and function in the RNAi pathway. To address the first issue, we asked whether affinitypurified TbDcll had the ability to digest dsRNA into siRNA-size molecules. To this end, we generated a cell line, termed BB2-TbDcl1, in which one of the two Dcl1 alleles was knocked out by replacement with a hygromycinselectable marker and the other one was tagged at the amino terminus with a BB2 epitope. The expression of the tagged protein was verified by Western blot analysis with anti-BB2 antibodies (Fig. 3A). Next, BB2-TbDcl1 was affinity selected from a cytoplasmic extract using protein G-Sepharose beads loaded with the BB2 monoclonal antibody. As a control, protein G-Sepharose beads without the antibody were similarly processed in the extract. Finally, the beads were incubated with homogeneously labeled dsRNA, and the products of digestions were separated by electrophoresis on a $15 \%$ sequencing gel (Fig. 3B). By this analysis, the beads loaded with the BB2 antibody (Fig. 3B,
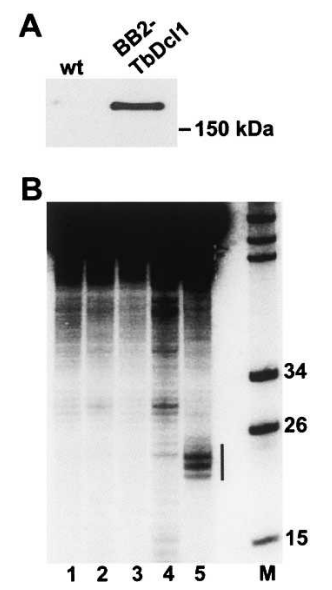

FIGURE 3. (A) Expression of BB2-tagged TbDcl1. Western blot of total cytoplasmic extracts from wild-type (wt) and BB2-TbDcll cells using the monoclonal BB2 antibody. (B) Affinity-purified BB2TbDcll has RNase III activity. BB2-TbDcll was affinity selected from a cytoplasmic extract with protein G-Sepharose-BB2 beads and incubated with radiolabeled dsRNA in digestion buffer (see Materials and Methods) containing magnesium (lane 5) or EDTA (lane 4), and the products of digestion were separated on a $15 \%$ sequencing gel. (Lane 1) Input dsRNA; (lanes 2,3) the BB2-TbDcll cytoplasmic extract was mock selected with protein G-Sepharose beads not loaded with antibody, and the beads were subsequently incubated with radiolabeled dsRNA in the presence of magnesium (lane 3) or 10 mM EDTA (lane 2). Note that most of the additional bands seen in lane 4 are also present in the input dsRNA and are better seen in lane 4 because a slightly larger quantity of dsRNA was added to the reaction. (M) End-labeled pBR322 MspI fragments used as molecular weight markers. The size in nucleotides of relevant fragments is indicated on the right. A bar indicates the small RNAs generated by affinity-purified TbDcllpreparations. The experiment was repeated twice with different preparations of dsRNA and protein, with similar results.

lane 5), but not the beads without antibodies (Fig. 3B, lane 3 ), captured an activity that generated RNA fragments of $\sim 24-26$ nt in length, which is the size distribution of endogenous T. brucei siRNAs (Djikeng et al. 2001). This activity was inhibited by the addition of EDTA to the reaction (Fig. 3B, cf. lanes 4 and 5). Thus, by this analysis, affinity-selected TbDcll copurified with a Dicer-like RNase III activity.

\section{RNAi down-regulation of TbDcl1 inhibits the RNAi response, the accumulation of endogenous siRNAs, and cleavage of dsRNA in vitro}

To further probe the function of TbDcll in the RNAi pathway, we down-regulated TbDcll expression by RNAi and examined whether the potency of the RNAi response was affected. This type of "catch 22" approach has been successfully used by others (Bernstein et al. 2001), but it should be noted that RNAi down-regulation of genes that are essential for RNAi can only result in partial suppression of the RNAi response. This is because the function of the gene that is down-regulated by RNAi is required for the 
RNAi response. Notwithstanding this limitation, we constructed an insect-form trypanosome cell line conditionally expressing dsRNA homologous to a portion of the TbDcll coding region. One representative clonal cell line, termed Dcl1-RNAi, was grown in the presence or absence of tet for $2 \mathrm{wk}$, and during this period of time we did not detect any effect on cell viability (data not shown). However, expression of Dcl1 dsRNA for $5 \mathrm{~d}$ resulted in $\sim 50 \%$ reduction in the steady-state amount of the corresponding Dcl1 mRNA (Fig. 4A). Next, we compared the ability of induced and uninduced Dcl1-RNAi cells to respond to transfection with dsRNA corresponding to the $5^{\prime}$-UTR of $\alpha$-tubulin mRNA (Ngo et al. 1998) by scoring for the presence of the socalled FAT phenotype (cells with multiple nuclei, flagella,
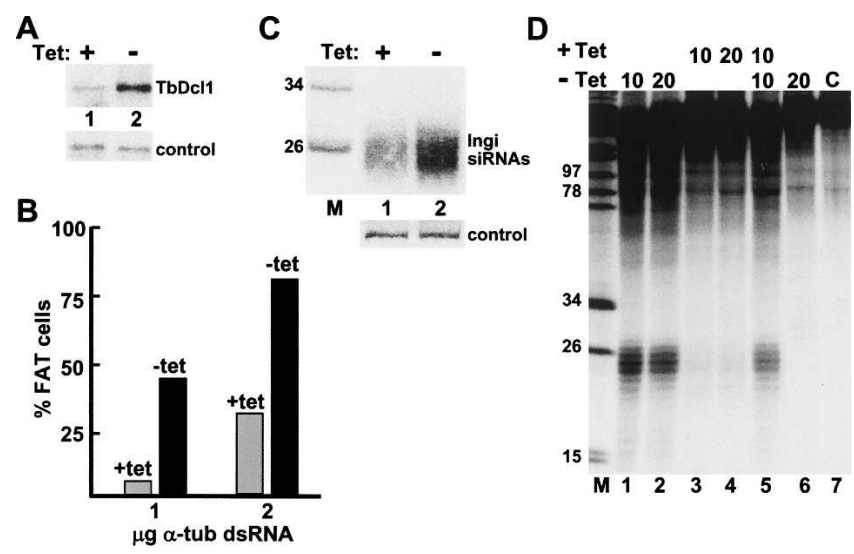

FIGURE 4. Down-regulation of TbDcll by RNAi. (A) Northern blot analysis of the steady-state level of TbDcll mRNA in Dcl1-RNAi cells grown in the presence $(+)$ or absence $(-)$ of tetracycline for $5 \mathrm{~d}$. (Control) Hybridization to the Piwi-likel mRNA as a loading control. Similar results were obtained with two independently isolated clonal cell lines. (B) Generation of FAT cells upon electroporation of $\alpha$-tubulin dsRNA. Dcll-RNAi cells were grown in the presence (gray bars) or absence (black bars) of tetracycline for $5 \mathrm{~d}$ and electroporated with the indicated amounts of $\alpha$-tubulin dsRNA. After $16 \mathrm{~h}$ of growth, the percentage of FAT cells (cells with multiple nuclei and flagella) was determined by observation at the optical microscope. Similar results were obtained with five independent clonal cell lines. $(C)$ The level of endogenous siRNAs derived from the retroposon Ingi is decreased in tet-induced Dcll-RNAi cells. Small RNAs were isolated from Dcl1-RNAi cells grown for $5 \mathrm{~d}$ in the presence $(+)$ or absence $(-)$ of tet and analyzed by Northern blot hybridization with an Ingi radiolabeled sense riboprobe. (Control) A cross-hybridizing band was used as a loading control; (M) molecular weight marker as in Figure 3B. The experiment was repeated twice with RNA isolated from two independent cell lines. (D) In vitro analysis of RNase III activity in extracts from Dcl1-RNAi cells. Cytoplasmic extracts were prepared from Dcl1-RNAi cells grown in the presence (+tet) or absence (-tet) of tet for $5 \mathrm{~d}$. Ten or $20 \mu \mathrm{L}$ of each extract, as indicated above each lane, were incubated in digestion buffer containing radiolabeled dsRNA, and the products of digestion were separated on a $15 \%$ sequencing gel. (Lane 7) Input dsRNA; (lane 6) as in lane 2, but with addition of 10 mM EDTA. (M) Molecular weight markers as in Figure 3B. The experiment was repeated three times with different extract preparations, different dsRNA, and different amounts of extracts. There was some variability in the amount of RNAse III activity in different extracts, but the results were always similar to those presented. and mitochondrial genomes, which arise because of the transient down-regulation of $\alpha$-tubulin). Uninduced Dcl1RNAi cells were very sensitive to dsRNA, with $\sim 45 \%$ and $85 \%$ of the cells becoming FAT after exposure to 1 and $2 \mu \mathrm{g}$ of dsRNA, respectively (Fig. 4B). In contrast, under identical transfection conditions, tet-induced Dcl1-RNAi cells produced $\sim 5 \%$ and $35 \%$ FAT cells in response to 1 and $2 \mu \mathrm{g}$ of dsRNA, respectively. Thus, there was considerable inhibition of the RNAi response at both dsRNA concentrations tested. In contrast, knockdown of Tb948 by RNAi did not affect the RNAi response (data not shown).

To gather further evidence for a role of TbDcl1 in the RNAi pathway, two additional parameters were compared in uninduced and tet-induced Dcl1-RNAi cells, namely, the steady-state levels of endogenous retroposon Ingi siRNAs (Djikeng et al. 2001; Shi et al. 2004a) and the ability of cell extracts to digest dsRNA to siRNA-size molecules. In the first experiment, the amount of siRNAs derived from the retroposon Ingi was evaluated by Northern blot analysis, which revealed that the accumulation of Ingi siRNAs was reduced $\sim 50 \%$ following Dcll down-regulation for $5 \mathrm{~d}$ (Fig. 4C). In the second experiment, we assessed the ability of cytoplasmic extracts from uninduced or 5-d induced Dcl1-RNAi cells to produce siRNA-size fragments from homogeneously labeled dsRNA. RNA fragments, 24-26 nt long, were readily detectable after incubation of the input dsRNA in the extract prepared from uninduced cells (Fig. $4 \mathrm{D}$, lanes 1,2$)$. In addition, we noticed the accumulation of a series of higher-molecular-weight RNA fragments with sizes of $\sim 80 \mathrm{nt}, 100 \mathrm{nt}$, and larger. In contrast, the extract prepared from induced cells was much less active at producing siRNA-size molecules, as well as higher-molecular-weight fragments, although some residual activity could still be detected (Fig. 4D, lanes 3,4). Mixing equal amounts of the two extracts also resulted in the appearance of small RNAs and higher-molecular-weight fragments (Fig. 4D, lane 5), suggesting that the reduced activity of the tet-induced cell extract was not due to the presence of a soluble inhibitor. As RNase III enzymes require divalent ions for activity, we added EDTA to the reaction containing extract from uninduced cells (Fig. 4D, lane 6) and verified that chelating magnesium reduced the accumulation of the small RNA fragments to undetectable levels. However, we did not find that addition of ATP had a stimulatory effect in the crude extract (data not shown), as reported by others (Best et al. 2005). Since we did not attempt to deplete ATP from our extracts, it is possible that ATP was not limiting.

\section{Overexpression of a mutant TbDcl1 inhibits the RNAi response}

Since several attempts to knock out (KO) both Dcl1 alleles failed, we decided to establish a cell line for conditional $\mathrm{KO}$ of DCl1. As a first step toward this goal, we inserted in the genome of wild-type 29.13.6 trypanosomes (Wirtz et al. 
1999) a tet-inducible ectopic copy of TbDcll carrying a dual BB2/FLAG epitope tag at the amino terminus (Fig. $5 \mathrm{~A})$. The resulting cell line, termed Dcl1-m, was incubated with increasing amounts of tet, and Western blot analysis with anti-BB2 antibodies confirmed expression of the tagged protein (Fig. 5B). In particular, tet concentrations of 0.1 and $1.0 \mu \mathrm{g} / \mathrm{mL}$ produced amounts of Dcl1 that were well above what is detectable in a cell line harboring a single tagged Dcl1 allele at its chromosomal locus (Fig. 5B, cf. lanes 4,5 and lane 6). It is worth noting that the level of TbAgol was not detectably affected when Dcl1 was overexpressed (Fig. 5B). Surprisingly, Dcl1-m cells induced with $1 \mu \mathrm{g} / \mathrm{mL}$ tet, and thus overexpressing the ectopic Dcl1 copy, showed $\sim 10$-fold reduction in the acquisition of the FAT phenotype following $\alpha$-tubulin dsRNA transfection, when compared to uninduced cells (Fig. 5C). A possible explanation for this unexpected result was revealed when we sequenced the BB2/FLAG-Dcl1-m cassette: compared to the gene sequence present in our Trypanosoma brucei rhodesiense laboratory strain, the ectopic copy had a single nucleotide substitution, which changed amino acid 966 from aspartate to glycine (D966G, indicated by a vertical

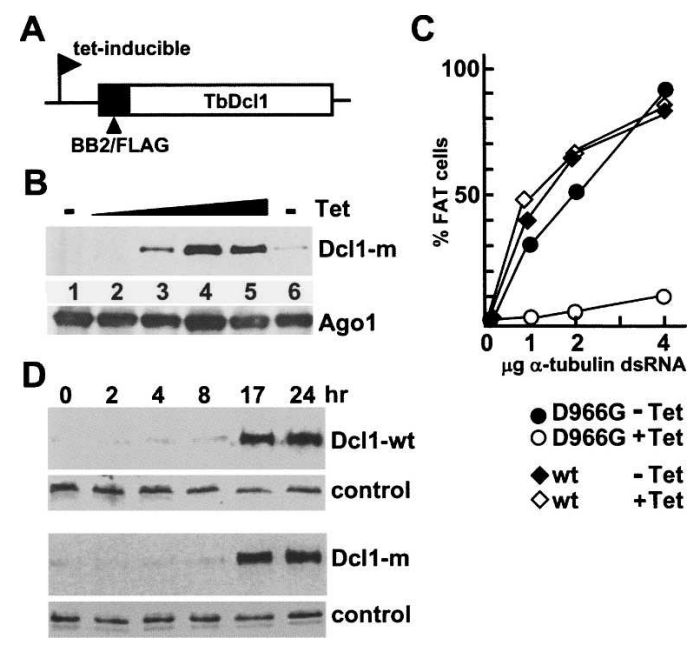

FIGURE 5. Inhibition of the RNAi response following overexpression of TbDcll carrying the D966G mutation. (A) Schematic representation of the BB2-FLAG-Dcl1 construct used for overexpression. "Tet-inducible" indicates the PARP promoter of plasmid vector pLEW79. (B) Expression of BB2/FLAG-Dcl-m. Dcl1-m cells were grown for $2 \mathrm{~d}$ in the absence (lane 1) or presence (lanes 2-5) of increasing concentrations of tet: (lane 2) $0.001 \mu \mathrm{g} / \mathrm{mL}$; (lane 3) 0.01 $\mu \mathrm{g} / \mathrm{mL}$; (lane 4) $0.1 \mu \mathrm{g} / \mathrm{mL}$; (lane 5) $1.0 \mu \mathrm{g} / \mathrm{mL}$. Total cell extracts were prepared and analyzed by Western blotting with the BB2 monoclonal antibody (upper panel). (Lane 6) Extract from an equivalent number of BB2-TbDcll cells expressing the tagged gene from the endogenous chromosomal locus. As a control, the membrane was stripped and reacted with a rabbit antiserum against TbAgol (lower panel). (C) FAT cell assay as described in Figure 4B. Dcll-m and Dcl1-wt cells were grown in the presence $(+$ Tet) or absence (-Tet) of tet for $4 \mathrm{~d}$ before being transfected with $\alpha$-tubulin dsRNA. (D) Dcll-wt and Dcl1-m cells were induced with $1.0 \mu \mathrm{g} / \mathrm{mL}$ tet for the time indicated above each lane, and total cell extracts were probed with anti-BB2 antibodies. (Control) Indicates a cross-reactive protein. bar in Fig. 1A). This residue is located $\sim 130$ amino acids downstream from the RNase IIIb motif, in a region of the protein devoid of recognizable domains or motifs. Nevertheless, Asp966 is conserved among T. brucei, T. vivax, and T. congolense Dcl1 genes. To examine whether the D966G mutation was the underlying cause for the observed inhibition of the RNAi response, we constructed a control cell line in which an ectopic wild-type copy of the BB2/FLAGDcll cassette was inserted in the genome under the control of a tet-responsive promoter (Dcl1-wt cells). Next, tetracycline $(1 \mu \mathrm{g} / \mathrm{mL})$ was added to Dcl1-wt and Dcl1-m cells, and protein expression of wild-type and D966G Dcl1 was monitored by Western blotting during a 24 -h period (Fig. 5D). This did not reveal a noticeable difference in the induction kinetics, and the two proteins accumulated to comparable levels after $17 \mathrm{~h}$ and $24 \mathrm{~h}$. However, contrary to what we noticed with D966G Dcl1, overexpression of the wt Dcll ectopic copy did not affect the RNAi response at a detectable level, as measured by the acquisition of the FAT phenotype (Fig. 5C). To corroborate the effect of the D966G mutation on the RNAi response, we next directly examined whether overexpression of the mutated protein affected the ability of trypanosomes to degrade target mRNA upon transfection of dsRNA. Thus, uninduced and tet-induced Dcl1-m cells were transfected with increasing amounts of $\alpha$-tubulin dsRNA, the extent of degradation of $\alpha$-tubulin mRNA was monitored by Northern blot analysis, and the results were quantitated by PhosphorImager analysis (Fig. 6A,B). Consistent with the observed FAT cell phenotype, tubulin mRNA was efficiently targeted in uninduced cells, but overexpression of D966G Dcl1 led to a two- to fivefold inhibition of $\alpha$-tubulin mRNA degradation.

Taken together, the above results are consistent with the hypothesis that the D966G mutation displays a dominantnegative effect. Unfortunately, the absence of recognizable domains surrounding Asp966 makes it at present impossible to speculate what step(s) in the RNAi pathway is(are) affected by overexpressing D966G Dcl1. What we know so far is that overexpression of the mutant protein does not affect the level of endogenous retroposon-derived siRNAs. As can be seen in Figure 6C, Northern blot analysis did not reveal a detectable difference in the steady-state level of Ingi siRNAs in uninduced and tet-induced Dcl1-m cells, suggesting that the D966G mutation does not have an effect on the processing of siRNAs from dsRNA.

\section{DISCUSSION}

Here we have used a combination of approaches to first identify and then characterize a Dicer-like protein that fuels the RNAi pathway in T. brucei. Our data indicate that the TbDcl1 gene fulfills the criteria for being the trypanosome Dicer homolog. First, TbDcll is restricted to RNAicompetent trypanosomatids, accumulates in the cytoplasm, 
A

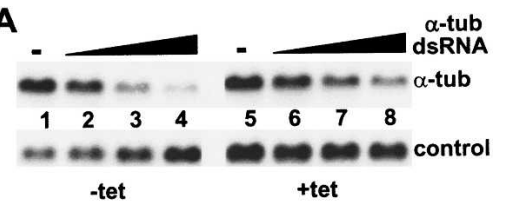

B

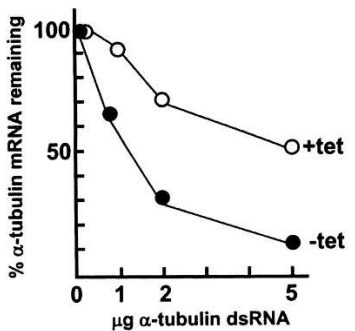

C

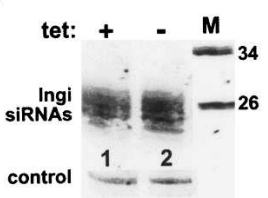

FIGURE 6. (A) Disappearance of $\alpha$-tubulin mRNA upon transfection with homologous dsRNA. TbDcl1-m cells were grown in the presence (+tet) or absence (-tet) of tet for $2 \mathrm{~d}$ and then electroporated with increasing amounts of $\alpha$-tubulin dsRNA representing the mRNA 5'-UTR: (lanes 2,6) $1.0 \mu \mathrm{g}$; (lanes 3,7) $2.0 \mu \mathrm{g}$; (lanes 4,8) $5.0 \mu \mathrm{g}$. (Lanes 1,5) Cells were electroporated with polyriboinosinicpolyribocytidylic acid [poly(I:C)]. Total RNA was prepared after $2 \mathrm{~h}$ and analyzed by Northern blotting using an $\alpha$-tubulin DNA probe representing a portion of the tubulin coding region. The bottom panel (control) shows the hybridization to the $12 \mathrm{~S}$ mitochondrial rRNA that served as a loading control. (B) Quantitation of the results shown in A. $\alpha$-Tubulin mRNA hybridization was quantitated by PhosphorImager analysis, normalized relative to the hybridization of the $12 \mathrm{~S}$ band, and plotted as the fraction of mRNA remaining $2 \mathrm{~h}$ after electroporation, taking as $100 \%$ the amount of $\alpha$-tubulin mRNA present in the samples that received poly $(I: C)$. (C) Analysis of the accumulation of endogenous Ingi siRNAs. TbDcll-m cells were grown in the presence $(+)$ or absence $(-)$ of tet for $2 \mathrm{~d}$, and the level of Ingi siRNAs was determined as described in the legend to Figure 4C. The experiments described in Figures 5 and 6 were repeated at least three times with similar outcomes.

and is endowed with two RNase III-like domains. Second, affinity-purified TbDcll generated siRNA-size molecules from long dsRNA. Third, both down-regulation of TbDcl1 by RNAi and overexpression of a mutant protein indicated that TbDcll function is required for the processing of dsRNA to siRNA-size molecules and for the RNAi pathway.

Whereas primary sequence searches revealed the presence of only one RNase III domain (RNase IIIb) in TbDcl1, further domain searches discovered that the T. vivax Dcll homolog had a second recognizable RNase III-like domain close to the amino terminus (RNase IIIa), and with this lead a similar domain could be recognized in TbDcll. As we noted, primary sequence searches failed to recognize the RNase IIIa motif in TbDcll, because the similarity with the prokaryotic RNase III consensus sequence was below the default threshold of significance. Besides being a large protein with two RNase III-like domains, analysis of the sequence of TbDcll by various domain search algorithms turned out to be uninformative. Compared to the structure of known Dicer enzymes from animals, plants, fungi, and the protist Giardia (Cerutti and Casas-Mollano 2006), T. brucei Dcll appears devoid of recognizable helicase, DUF83, PAZ, and dsRBD domains. However, this apparent domain deficiency needs to be interpreted with caution, since search algorithms are of limited value when the primary sequence of a domain is highly divergent, as it may be the case in TbDcll. Only structural analysis of TbDcll will offer insight into its domain structure. On the other hand, it is evident from the positions of the RNase IIIa and RNase IIIb motifs, relative to each other and to the protein termini, that TbDcll is significantly different from Dicer enzymes of other organisms. Specifically, whereas in most Dicers, the two RNase III domains are located toward the carboxyl terminus, in TbDcll, one is close to the amino terminus and the other is centrally located.

According to the CLUSTALW alignment of Figure 2A, the RNase IIIa motif of TbDcll is rather unusual, because it is missing acidic residues at positions equivalent to the A. aeolicus RNase III residues 37, 40, and 110 . In particular, glutamate 110 is essential for catalysis both in bacterial RNase III and in human Dicer (Zhang et al. 2004; Gan et al. 2006). However, one should note that in the T. brucei RNase IIIa domain, there are two glutamate residues in the vicinity of glutamate 110, one at position 105 and a second one at position 107, and that the sequences preceding and following position 110 can only be poorly aligned. Thus, whether the TbDcll RNase IIIa motif has a residue functioning as the equivalent of glutamate 110 is unclear. Also, in favor of the RNase IIIa motif being a bona fide RNase III domain is the structure model prediction (Fig. 2B), suggestive that the main structural features of RNase III are still present in this domain.

Down-regulation of TbDcl1 expression by RNAi resulted in a significant diminution of the RNAi response and $\sim 50 \%$ decrease in the steady-state level of endogenous siRNAs. Furthermore, extracts from tet-induced TbDcl1RNAi cells displayed a severe reduction in the major RNase III activity that generates siRNA-size fragments from long dsRNAs (Best et al. 2005). These observations, in combination with the Dicer-like activity copurifying with BB2tagged Dcl1, are consistent with TbDcl1 representing the enzyme that processes dsRNA to generate siRNAs.

Unexpectedly, in wild-type cells, ectopic overexpression of a mutant TbDcl1, carrying a substitution at position 966 of a conserved aspartate with glycine, resulted in inhibition of the RNAi response, as assayed by the ability of the cells to respond to transfection with $\alpha$-tubulin dsRNA. However, inhibition of the RNAi response was not concomitant with a diminution in the steady-state level of endogenous siRNAs. Since endogenous siRNAs have a half-life of $\sim 12 \mathrm{~h}$ in T. brucei (H. Shi, A. Djikeng, and E. Ullu, unpubl.), the unchanged levels of endogenous siRNAs indicate that the 
D966G mutation is likely not affecting the ability of TbDcl1 to generate siRNAs from endogenous retroposon dsRNA. However, whether or not the D966G mutation has any effect on the catalytic activity of TbDcll can only be addressed with recombinant or highly purified enzyme, and will be the subject of future experiments. Acknowledging the limitation of our present understanding of the precise effect(s) of the D966G mutation, the dominantnegative effect of the mutation nevertheless suggests that TbDcl1 interacts with other components of the RNAi pathway. Guided by current evidence from other systems, likely candidates for interaction with TbDcll are TbAgol (Tahbaz et al. 2004), RISC itself (Lee et al. 2004; Pham et al. 2004), a homolog of Drosophila R2D2 (Liu et al. 2003), or a homolog of human TRBP (Chendrimada et al. 2005; Haase et al. 2005). Unfortunately, so far we have been unable to capture a stable interaction between TbDcll and TbAgo1. In addition, there are no other known components of RISC besides TbAgo1, and the T. brucei genome does not contain obvious R2D2 or TRBP homologs. Notwithstanding the uncertainty about its role, the inhibitory effect of the D966G mutation on the RNAi response lends further support to the identification of TbDcll as the trypanosome Dicer homolog.

In conclusion, our data indicate that TbDcll is a highly divergent Dicer family member. This is quite surprising considering that the domain structure of the Giardia Dicer (Macrae et al. 2006) is similar to that of higher eukaryotic Dicers, although being much smaller in size and lacking the helicase domain. It has been argued that the similarity of Giardia Dicer to higher eukaryotic Dicers indicates that all Dicers evolved from a common ancestral enzyme (Macrae et al. 2006). However, recent phylogenetic analyses did not resolve the relationship between most Dicer and Dicer-like proteins (Cerutti and Casas-Mollano 2006). Only the animal and plant Dicer and Dicer-like genes formed a well-defined cluster suggestive of a monophyletic origin. As suggested in Drosophila (Obbard et al. 2006), the variation in the structure of $\mathrm{Dcl}$ genes of protozoa may be the result of a coevolutionary "arms race" between RNAi-pathway genes and the endogenous RNAi target genes, such as viruses and mobile elements.

\section{MATERIALS AND METHODS}

\section{Trypanosome cell lines}

Procyclic cells of strain T. brucei rhodesiense Ytat1.1 were transfected as previously described (Shi et al. 2004a). For expression of Tb1648-gfp and Tb948-gfp, cells were transiently transfected with appropriate constructs made in the plasmid vector pXSGFPM3FUS (Marchetti et al. 2000). Knockout of one of the two TbDcll alleles was carried out by homologous recombination with a PCR-assembled cassette in which the TbDcll coding region was replaced with the hygromycin antibiotic selectable marker
(Arhin et al. 2004). Tagging of the second Dcll allele with the BB2 epitope was carried out using the blasticidin-resistance marker (BB2-TbDcll cell line). For RNAi down-regulation of TbDcll nucleotides 4-500 of the coding region were inserted between the two opposing tet-inducible T7 RNA polymerase promoters of plasmid vector pZJM carrying the phleomycin-resistance marker (Wang et al. 2000). The construct was linearized with NotI for integration at the ribosomal DNA nontranscribed spacer region of procyclic strain 29.13.6, expressing the tet repressor and T7 RNA polymerase (Wirtz et al. 1999).

\section{Antibodies, immunoprecipitations, and Western blot analysis}

BB2 monoclonal antibodies recognize an epitope tag corresponding to 10 amino acids from the major structural protein of the Saccharomyces cerevisiae Tyl virus-like particle (Bastin et al. 1996). Anti-BB2 antibodies bound to protein G-Sepharose beads in NET-2 buffer (150 mM NaCl, $50 \mathrm{mM}$ Tris at $\mathrm{pH} 7.5,0.05 \%$ NP-40) were mixed with cell extracts and incubated for $4 \mathrm{~h}$ at $4^{\circ} \mathrm{C}$. The beads were then washed seven times with NET-2, and equivalent amounts of supernatants and beads were used for Western blot analysis or for RNase III activity assays. Western blots were developed using ECL reagents (Amersham Biosciences) and exposed to Kodak XAR film.

\section{RNase III assays}

For the preparation of dsRNA, we used plasmid p2T $\alpha$ tub-113 (nucleotides 1-113 of $\alpha$-tubulin mRNA) or p2T $\alpha$ tub-170 (nucleotides $1-170$ of $\alpha$-tubulin mRNA), which are derivatives of plasmid p2T containing opposing T7 RNA polymerase promoters (Shi et al. 2000). The plasmids were linearized with an appropriate restriction enzyme and used as templates to generate radiolabeled sense or antisense strand transcripts using the Ambion Megashort Script kit. Reactions contained $1.5 \mu \mathrm{g}$ of DNA, $75 \mu \mathrm{Ci}$ of $[\alpha-$ ${ }^{32} \mathrm{P}$ ]UTP $(3000 \mathrm{Ci} / \mathrm{mmol})$, and $18.75 \mu \mathrm{M}$ UTP. The remainder of the components were according to the manufacturer's instructions. After digestion with DNase I and removal of unincorporated NTPs on a P6 column (Bio-Rad), equimolar amounts of the sense and antisense transcripts were mixed, heated for $1 \mathrm{~min}$ at $90^{\circ} \mathrm{C}$, annealed in $300 \mathrm{mM} \mathrm{NaCl}, 20 \mathrm{mM}$ Tris- $\mathrm{HCl}$ (pH 7.5), and $5 \mathrm{mM}$ EDTA for $1 \mathrm{~h}$ at $65^{\circ} \mathrm{C}$, and then allowed to cool to room temperature. Remaining single-stranded RNA was digested with 5 units of ribonuclease $\mathrm{T} 1$ for $30 \mathrm{~min}$ on ice. The RNA was phenol-extracted, precipitated with ethanol, resuspended in $20 \mu \mathrm{L}$ of urea loading dye ( $7 \mathrm{M}$ urea in $1 \times \mathrm{TBE}$ ) at $37^{\circ} \mathrm{C}$, and fractionated on a $6 \%$ sequencing gel. dsRNA was eluted from the gel slice in $0.5 \mathrm{M} \mathrm{Na}$-acetate overnight at room temperature, recovered by ethanol precipitation, and resuspended in water.

For assaying RNase III activity in crude extracts, cytoplasmic extracts were prepared as previously described (Gunzl et al. 1995), spun at $200,000 \mathrm{~g}$ for $10 \mathrm{~min}$, and frozen in aliquots or used immediately. The protein concentration of the extracts was determined by absorbance at $280 \mathrm{~nm}$. The $100 \mu \mathrm{L}$ reactions contained 10 or $20 \mu \mathrm{L}$ of extract, $20 \mathrm{mM} \mathrm{K}$-glutamate, $20 \mathrm{mM}$ Tris-HEPES (pH 7.2), $8 \mathrm{mM} \mathrm{MgCl}, 10 \mu \mathrm{g} / \mathrm{mL}$ leupeptin, $1 \mathrm{mM} \mathrm{DTT}$, and $\sim 100,000 \mathrm{cpm}$ of radiolabeled dsRNA. After addition of the extract, the reactions were incubated for $2 \mathrm{~h}$ at $28^{\circ} \mathrm{C}$ and phenol extracted. The RNase III activity of affinity-purified BB2-TbDcl1 was assayed as previously described (Zhang et al. 2002). 


\section{Other procedures}

RNA extraction, dsRNA transfection, and Northern blot analysis were performed as described previously (Shi et al. 2004a).

\section{ACKNOWLEDGMENTS}

We are grateful to Kristin Patrick for help with the Northern blot of Figure 4B and for critical reading of the manuscript. This work was supported by Public Health Service grants AI28798 to E.U. from NIAID.

Received July 30, 2006; accepted September 6, 2006.

\section{REFERENCES}

Arhin, G.K., Shen, S., Ullu, E., and Tschudi, C. 2004. A PCR-based method for gene deletion and protein tagging in Trypanosoma brucei. Methods Mol. Biol. 270: 277-286.

Bastin, P., Bagherzadeh, Z., Matthews, K.R., and Gull, K. 1996. A novel epitope tag system to study protein targeting and organelle biogenesis in Trypanosoma brucei. Mol. Biochem. Parasitol. 77: 235-239.

Baumberger, N. and Baulcombe, D.C. 2005. Arabidopsis ARGONAUTE1 is an RNA Slicer that selectively recruits microRNAs and short interfering RNAs. Proc. Natl. Acad. Sci. 102: 11928-11933.

Bernstein, E., Caudy, A.A., Hammond, S.M., and Hannon, G.J. 2001. Role for a bidentate ribonuclease in the initiation step of RNA interference. Nature 409: 363-366.

Best, A., Handoko, L., Schluter, E., and Goringer, H.U. 2005. In vitro synthesized small interfering RNAs elicit RNA interference in African trypanosomes: An in vitro and in vivo analysis. J. Biol. Chem. 280: 20573-20579.

Blaszczyk, J., Tropea, J.E., Bubunenko, M., Routzahn, K.M., Waugh, D.S., Court, D.L., and Ji, X. 2001. Crystallographic and modeling studies of RNase III suggest a mechanism for doublestranded RNA cleavage. Structure 9: 1225-1236.

Carmell, M.A. and Hannon, G.J. 2004. RNase III enzymes and the initiation of gene silencing. Nat. Struct. Mol. Biol. 11: 214-218.

Cerutti, H. and Casas-Mollano, J.A. 2006. On the origin and functions of RNA-mediated silencing: From protists to man. Curr. Genet. 50: 81-99.

Chendrimada, T.P., Gregory, R.I., Kumaraswamy, E., Norman, J., Cooch, N., Nishikura, K., and Shiekhattar, R. 2005. TRBP recruits the Dicer complex to Ago2 for microRNA processing and gene silencing. Nature 436: 740-744.

DaRocha, W.D., Otsu, K., Teixeira, S.M., and Donelson, J.E. 2004. Tests of cytoplasmic RNA interference (RNAi) and construction of a tetracycline-inducible T7 promoter system in Trypanosoma cruzi. Mol. Biochem. Parasitol. 133: 175-186.

Djikeng, A., Shi, H., Tschudi, C., and Ullu, E. 2001. RNA interference in Trypanosoma brucei: Cloning of small interfering RNAs provides evidence for retroposon-derived 24-26-nucleotide RNAs. RNA 7: 1522-1530.

Durand-Dubief, M. and Bastin, P. 2003. TbAGO1, an Argonaute protein required for RNA interference is involved in mitosis and chromosome segregation in Trypanosoma brucei. BMC Biol. 1: 2.

Gan, J., Tropea, J.E., Austin, B.P., Court, D.L., Waugh, D.S., and Ji, X. 2006. Structural insight into the mechanism of double-stranded RNA processing by ribonuclease III. Cell 124: 355-366.

Gunzl, A., Tschudi, C., Nakaar, V., and Ullu, E. 1995. Accurate transcription of the Trypanosoma brucei U2 small nuclear RNA gene in a homologous extract. J. Biol. Chem. 270: 17287 17291.

Haase, A.D., Jaskiewicz, L., Zhang, H., Laine, S., Sack, R., Gatignol, A., and Filipowicz, W. 2005. TRBP, a regulator of cellular PKR and
HIV-1 virus expression, interacts with Dicer and functions in RNA silencing. EMBO Rep. 6: 961-967.

Inoue, N., Otsu, K., Ferraro, D.M., and Donelson, J.E. 2002. Tetracycline-regulated RNA interference in Trypanosoma congolense. Mol. Biochem. Parasitol. 120: 309-313.

Lee, Y.S., Nakahara, K., Pham, J.W., Kim, K., He, Z., Sontheimer, E.J., and Carthew, R.W. 2004. Distinct roles for Drosophila Dicer-1 and Dicer-2 in the siRNA/miRNA silencing pathways. Cell 117: 69-81.

Liu, Q., Rand, T.A., Kalidas, S., Du, F., Kim, H.E., Smith, D.P., and Wang, X. 2003. R2D2, a bridge between the initiation and effector steps of the Drosophila RNAi pathway. Science 301: 1921-1925.

Liu, J., Carmell, M.A., Rivas, F.V., Marsden, C.G., Thomson, J.M., Song, J.J., Hammond, S.M., Joshua-Tor, L., and Hannon, G.J. 2004. Argonaute2 is the catalytic engine of mammalian RNAi. Science 305: 1437-1441.

Macrae, I.J., Zhou, K., Li, F., Repic, A., Brooks, A.N., Cande, W.Z., Adams, P.D., and Doudna, J.A. 2006. Structural basis for doublestranded RNA processing by Dicer. Science 311: 195-198.

Marchetti, M.A., Tschudi, C., Kwon, H., Wolin, S.L., and Ullu, E. 2000. Import of proteins into the trypanosome nucleus and their distribution at karyokinesis. J. Cell Sci. 113: 899-906.

Meister, G., Landthaler, M., Patkaniowska, A., Dorsett, Y., Teng, G., and Tuschl, T. 2004. Human Argonaute2 mediates RNA cleavage targeted by miRNAs and siRNAs. Mol. Cell 15: 185-197.

Miyoshi, K., Tsukumo, H., Nagami, T., Siomi, H., and Siomi, M.C. 2005. Slicer function of Drosophila Argonautes and its involvement in RISC formation. Genes \& Dev. 19: 2837-2848.

Mochizuki, K. and Gorovsky, M.A. 2005. A Dicer-like protein in Tetrahymena has distinct functions in genome rearrangement, chromosome segregation, and meiotic prophase. Genes \& Dev. 19: 77-89.

Ngo, H., Tschudi, C., Gull, K., and Ullu, E. 1998. Double-stranded RNA induces mRNA degradation in Trypanosoma brucei. Proc. Natl. Acad. Sci. 95: 14687-14692.

Obbard, D.J., Jiggins, F.M., Halligan, D.L., and Little, T.J. 2006. Natural selection drives extremely rapid evolution in antiviral RNAi genes. Curr. Biol. 16: 580-585.

Panigrahi, A.K., Ernst, N.L., Domingo, G.J., Fleck, M., Salavati, R., and Stuart, K.D. 2006. Compositionally and functionally distinct editosomes in Trypanosoma brucei. RNA 12: 1038-1049.

Pham, J.W., Pellino, J.L., Lee, Y.S., Carthew, R.W., and Sontheimer, E.J. 2004. A Dicer-2-dependent 80s complex cleaves targeted mRNAs during RNAi in Drosophila. Cell 117: 83-94.

Provost, P., Dishart, D., Doucet, J., Frendewey, D., Samuelsson, B., and Radmark, O. 2002. Ribonuclease activity and RNA binding of recombinant human Dicer. EMBO J. 21: 5864-5874.

Rivas, F.V., Tolia, N.H., Song, J.J., Aragon, J.P., Liu, J., Hannon, G.J., and Joshua-Tor, L. 2005. Purified Argonaute2 and an siRNA form recombinant human RISC. Nat. Struct. Mol. Biol. 12: 340-349.

Robinson, K.A. and Beverley, S.M. 2003. Improvements in transfection efficiency and tests of RNA interference (RNAi) approaches in the protozoan parasite Leishmania. Mol. Biochem. Parasitol. 128: 217-228.

Shi, H., Diikeng, A., Mark, T., Wirtz, E., Tschudi, C., and Ullu, E. 2000. Genetic interference in Trypanosoma brucei by heritable and inducible double-stranded RNA. RNA 6: 1069-1076.

Shi, H., Djikeng, A., Tschudi, C., and Ullu, E. 2004a. Argonaute protein in the early divergent eukaryote Trypanosoma brucei: Control of small interfering RNA accumulation and retroposon transcript abundance. Mol. Cell. Biol. 24: 420-427.

Shi, H., Ullu, E., and Tschudi, C. 2004b. Function of the trypanosome Argonaute 1 protein in RNA interference requires the N-terminal RGG domain and arginine 735 in the Piwi domain. J. Biol. Chem. 279: 49889-49893.

Shi, H., Tschudi, C., and Ullu, E. 2006. Functional replacement of Trypanosoma brucei Argonaute by the human slicer Argonaute2. RNA 12: 943-947.

Tahbaz, N., Kolb, F.A., Zhang, H., Jaronczyk, K., Filipowicz, W., and Hobman, T.C. 2004. Characterization of the interactions between 
mammalian PAZ PIWI domain proteins and Dicer. EMBO Rep. 5: 189-194.

Tomari, Y. and Zamore, P.D. 2005. Perspective: Machines for RNAi. Genes \& Dev. 19: 517-529.

Wang, Z., Morris, J.C., Drew, M.E., and Englund, P.T. 2000. Inhibition of Trypanosoma brucei gene expression by RNA interference using an integratable vector with opposing T7 promoters. J. Biol. Chem. 275: 40174-40179.

Wirtz, E., Leal, S., Ochatt, C., and Cross, G.A. 1999. A tightly regulated inducible expression system for conditional gene knock- outs and dominant-negative genetics in Trypanosoma brucei. Mol. Biochem. Parasitol. 99: 89-101.

Zamore, P.D. and Haley, B. 2005. Ribo-gnome: The big world of small RNAs. Science 309: 1519-1524.

Zhang, H., Kolb, F.A., Brondani, V., Billy, E., and Filipowicz, W. 2002. Human Dicer preferentially cleaves dsRNAs at their termini without a requirement for ATP. EMBO J. 21: 5875-5885.

Zhang, H., Kolb, F.A., Jaskiewicz, L., Westhof, E., and Filipowicz, W. 2004. Single processing center models for human Dicer and bacterial RNase III. Cell 118: 57-68. 

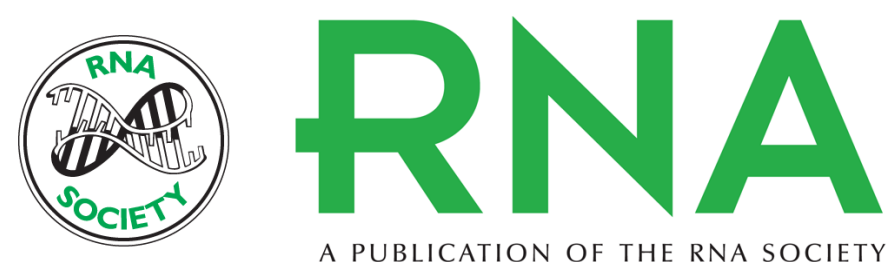

A PUBLICATION OF THE RNA SOCIETY

\section{An unusual Dicer-like1 protein fuels the RNA interference pathway in Trypanosoma brucei}

Huafang Shi, Christian Tschudi and Elisabetta Ullu

RNA 2006 12: 2063-2072 originally published online October 19, 2006

Access the most recent version at doi:10.1261/rna.246906

References This article cites 42 articles, 21 of which can be accessed free at:

http://rnajournal.cshlp.org/content/12/12/2063.full.html\#ref-list-1

License

Email Alerting Receive free email alerts when new articles cite this article - sign up in the box at the

Service top right corner of the article or click here.

To subscribe to RNA go to:

http://rnajournal.cshlp.org/subscriptions 